Full Wave Analysis of RF Signal Attenuation in a Lossy Rough Surface Cave using a High Order Time Domain Vector Finite Element Method

J. Pingenot, R. Rieben, D. White, D. Dudley

November 10, 2005

Progress in Electromagnetics Research (PIERS) 2006 Cambridge, MA, United States March 26, 2005 through March 29, 2005 
This document was prepared as an account of work sponsored by an agency of the United States Government. Neither the United States Government nor the University of California nor any of their employees, makes any warranty, express or implied, or assumes any legal liability or responsibility for the accuracy, completeness, or usefulness of any information, apparatus, product, or process disclosed, or represents that its use would not infringe privately owned rights. Reference herein to any specific commercial product, process, or service by trade name, trademark, manufacturer, or otherwise, does not necessarily constitute or imply its endorsement, recommendation, or favoring by the United States Government or the University of California. The views and opinions of authors expressed herein do not necessarily state or reflect those of the United States Government or the University of California, and shall not be used for advertising or product endorsement purposes. 


\title{
Full Wave Analysis of RF Signal Attenuation in a Lossy Rough Surface Cave using a High Order Time Domain Vector Finite Element Method*
}

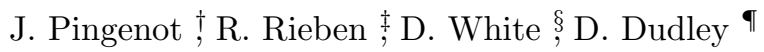

\begin{abstract}
We present a computational study of signal propagation and attenuation of a $200 \mathrm{MHz}$ planar loop antenna in a cave environment. The cave is modeled as a straight and lossy random rough wall. To simulate a broad frequency band, the full wave Maxwell equations are solved directly in the time domain via a high order vector finite element discretization using the massively parallel CEM code EMSolve. The numerical technique is first verified against theoretical results for a planar loop antenna in a smooth lossy cave. The simulation is then performed for a series of random rough surface meshes in order to generate statistical data for the propagation and attenuation properties of the antenna in a cave environment. Results for the mean and variance of the power spectral density of the electric field are presented and discussed.
\end{abstract}

\section{Introduction}

The study of electromagnetic wave propagation in caves and tunnels is of great practical interest to antenna engineers due to the increasing demands for reliable wireless communications systems in such environments. Current wireless radio frequency (RF) communication systems were not designed to operate reliably in enclosed environments such as caves and tunnels, and signal quality is severely compromised due to the rough and lossy surfaces of the cave. Today there is limited ability to maintain communications in cave-like structures, tunnels or subways, prohibiting the quick deployment of wireless systems in caves and tunnels. If the electromagnetic properties of the tunnel could be characterized (e.g. dissipation, dispersion, fading, etc.), then a more robust communication system could be designed specifically for operation in such environments, hence full wave EM simulations of propagation in this type of environment are very useful.

Much theoretical work in this field has been done in order to develop a better understanding of the RF propagation channel. Dudley recently studied models for propagation in lossy circular tunnels [1]. He produced expressions for the fields in terms of a Fourier transform over the axial variables, and presented the numerical results for the field intensity both as a function of axial distance and as a function of radial distance. However, this work only involved smooth tunnel walls and not the more realistic situation of rough wall tunnels. In this case, the electromagnetic fields are no longer deterministic, but random variables that need to be solved using statistical methods. Recently, Pao and Kasey have investigated the statistical properties of wave propagation in straight, rough-walled tunnels [2], [3]. This work assumes a perfect electrical conductor (PEC) boundary at the rough wall / air interface. A more realistic model needs to take into account the lossy nature of the rough walls and the cave material (typically granite or some sort of earth like material with electrical conductivities on the order of $0.1 S / m)$.

In this paper we use a high order finite element discretization to solve the full wave Maxwell equations directly in the time domain for the case of a planar loop antenna placed at the mouth of a straight, lossy rough walled tunnel. We chose a time domain simulation in order to efficiently compute the response over a broad frequency band. We begin with a brief description of the numerical method employed for this problem (as implemented in the EMSolve code [4]). We then verify the numerical method against the theoretical results of Dudley [1] for a planar loop antenna in a smooth lossy cave and discuss the limitations of the numerical model. Finally, we proceed to solve the electromagnetic wave equation on a sequence of randomly generated meshes to determine statistical properties for the power spectral density of the electric field.

${ }^{*}$ This work was performed under the auspices of the U.S. Department of Energy by the University of California, Lawrence Livermore National Laboratory under contract No. W-7405-Eng-48, UCRL-CONF-208481

${ }^{\dagger}$ University of Washington, pingej@u. washington.edu

¥Lawrence Livermore National Laboratory, rieben1@llnl.gov

$\S$ Lawrence Livermore National Laboratory, white37@llnl.gov

I University of Arizona, dudley@ece.arizona.edu 


\section{Numerical Formulation}

We begin with the second order time dependent wave equation for the electric field in a 3 dimensional domain $\Omega$

$$
\begin{aligned}
\epsilon \frac{\partial^{2}}{\partial t^{2}} \mathbf{E} & =-\nabla \times\left(\mu^{-1} \nabla \times \mathbf{E}\right)-\sigma \frac{\partial}{\partial t} \mathbf{E}-\frac{\partial}{\partial t} \mathbf{J} & & \text { in } \Omega \\
\nabla \cdot(\epsilon \mathbf{E}) & =0 & & \text { in } \Omega \\
\hat{\mathbf{n}} \times \mathbf{E} & =\mathbf{E}_{b c} & & \text { on } \partial \Omega
\end{aligned}
$$

where $\partial \Omega$ is the two dimensional boundary of the domain, $\hat{\mathbf{n}}$ is the outwardly directed unit normal of this boundary and $\mathbf{J}$ is a free current density source that can be added to drive the problem. The value $\mathbf{E}_{b c}$ represents an arbitrary boundary condition imposed on the electric field intensity while $\epsilon, \mu$ and $\sigma$ denote the dielectric permittivity, magnetic permeability and electrical conductivity of the materials contained in the domain $\Omega$ respectively.

Applying an arbitrary order Galerkin finite element discretization to the wave equation (the details of which can be found in [5], [6]) yields the following semi-discrete system of ordinary differential equations

$$
M_{\epsilon} \frac{\partial^{2}}{\partial t^{2}} e=-S_{\mu} e-M_{\sigma} \frac{\partial}{\partial t} e-\frac{\partial}{\partial t} j
$$

where $M_{\epsilon}, M_{\sigma}$ are finite element mass matrices, $S_{\mu}$ is a finite element stiffness matrix and $e, j$ are discrete arrays of finite element degrees of freedom. Applying a backward difference approximation for the first order time derivative in (2) and a central difference approximation for the second order time derivative yields the following fully discrete linear system of equations

$$
M_{\epsilon} e_{n+1}=\left(2 M_{\epsilon}-\Delta t^{2} S_{\mu}-\Delta t M_{\sigma}\right) e_{n}+\left(\Delta t M_{\sigma}-M_{\epsilon}\right) e_{n-1}-j^{\prime}
$$

where $\Delta t$ is the discrete time step, the integer $n$ denotes the current time step and the time derivative of the free current source has been directly incorporated into the new source term $j^{\prime}$.

For the results shown in section 4 below we employ second-order interpolatory $H(\mathrm{curl})$ basis functions along with custom quadrature rules that yield a diagonal "mass" matrix $M_{\epsilon}$. The details of this discretization are presented in [7]. This method is much more accurate than standard FDTD, the numerical dispersion for this method is $O\left(h^{4}\right)$ rather than $O\left(h^{2}\right)$ as it is for FDTD. Compared to higher-order FDTD schemes, this method is better at modeling the jump discontinuity of fields across the air-earth interface.

\section{Verification}

Before we proceed to simulations of a random rough surface, we begin by verifying our numerical method with known theoretical values for the case of a smooth, lossy tunnel. These results were computed by Dudley according to the procedures described in [1]. We consider the case of an axially symmetric circular current loop of radius $b=0.2 \mathrm{~m}$ placed at the mouth of a circular tunnel of length $60 \mathrm{~m}$ and radius $a=2.0 \mathrm{~m}$. The current loop is driven by a time harmonic source of frequency $f=200 \mathrm{MHz}$. The electric field is "measured" along the length of the tunnel at a radial observation point $\rho$ such that $\rho / a=0.3$ The tunnel has a relative dielectric constant of $\epsilon_{r}=5.0$. We consider two cases, a tunnel with an electric conductivity $\sigma_{\text {low }}=0.02 \mathrm{~S} / \mathrm{m}$ and $\sigma_{\text {high }}=0.1 \mathrm{~S} / \mathrm{m}$.

For the numerical model, we discretize the tunnel domain in two different ways using both a Cartesian (or "stair-step") approximation to the smooth tunnel wall and a more accurate conforming cylindrical mesh (see Figure 1 and Figure 2). For both cases, a planar loop of current of radius $0.2 m$ is placed at one end of the tunnel, while a simple absorbing boundary condition $(\mathrm{ABC})$ is placed at the other. The $\mathrm{ABC}$ is imperfect for anything other than plane waves at normal incidence, hence we make our tunnel mesh $75 \mathrm{~m}$ long and ignore field data from the last $20 \%$ of the tunnel mesh. A perfect electric conductor (PEC) boundary condition is applied to the outer most walls of the cave to fully define the problem. For both the Cartesian and Cylindrical meshes, the outermost PEC boundary is made sufficiently large to prevent spurious reflections. The temporal dependence of the current source is a Gaussian pulsed sine wave centered at $200 \mathrm{MHz}$ with a $20 \%$ bandwidth. The simulation is performed using high order $p=2$ basis functions to mitigate the effects of numerical dispersion.

In Figure 3 and Figure 4 we compare results for both numerical models (Cartesian and cylindrical meshes) to the theoretical results for both conductivity values. Note that the agreement between the theoretical model and the numerical model using the conforming cylindrical mesh are excellent, indicating that the proposed numerical method is working properly. The discrepancies between the Cartesian results and the theoretical results are due to 


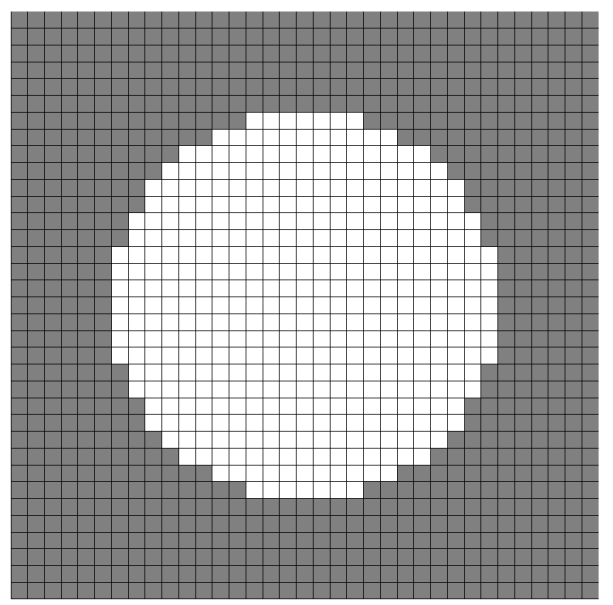

Figure 1: Cross section of smooth tunnel Cartesian mesh.

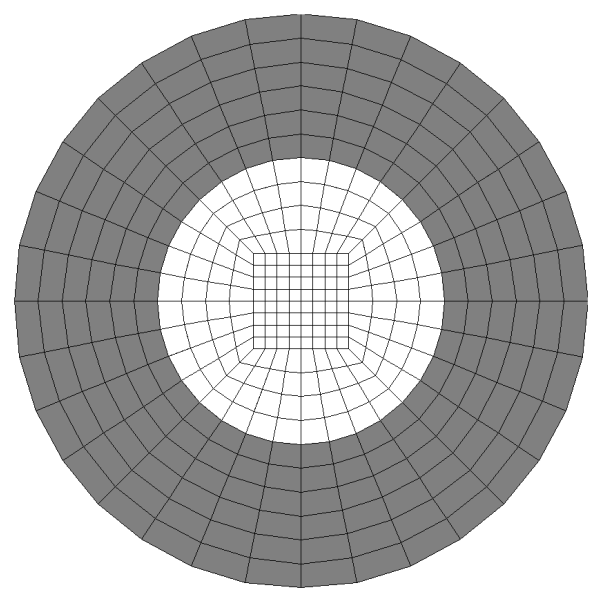

Figure 2: Cross section of smooth tunnel conforming cylindrical mesh.

the "stair-step" approximation to smooth surfaces, which is known to be problematic since such approximations fail to converge to a true cylindrical surface. While the Cartesian mesh is a bad choice for modeling smooth tunnels, it is sufficient for modeling rough surface tunnles, which we will use in the next section.

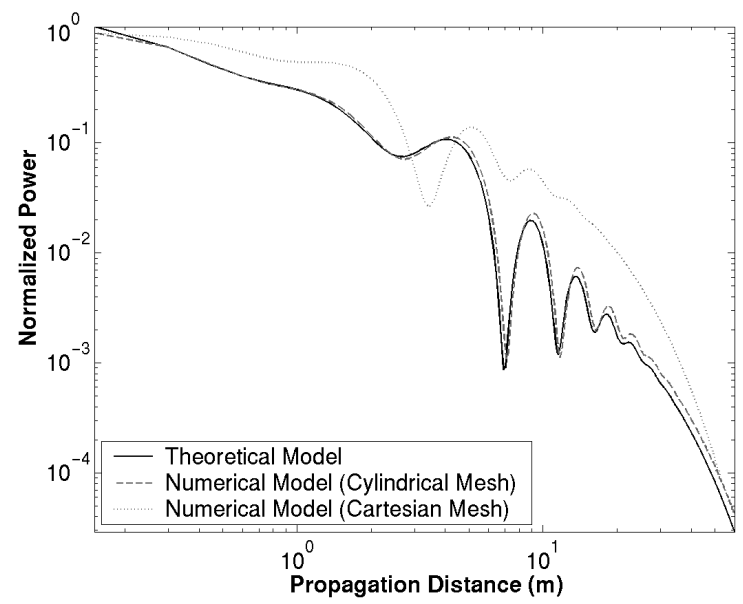

Figure 3: Comparison between theoretical model and two different numerical models at $200 \mathrm{MHz}$ for a smooth cave with conductivity $\sigma_{\text {low }}=0.02 \mathrm{~S} / \mathrm{m}$.

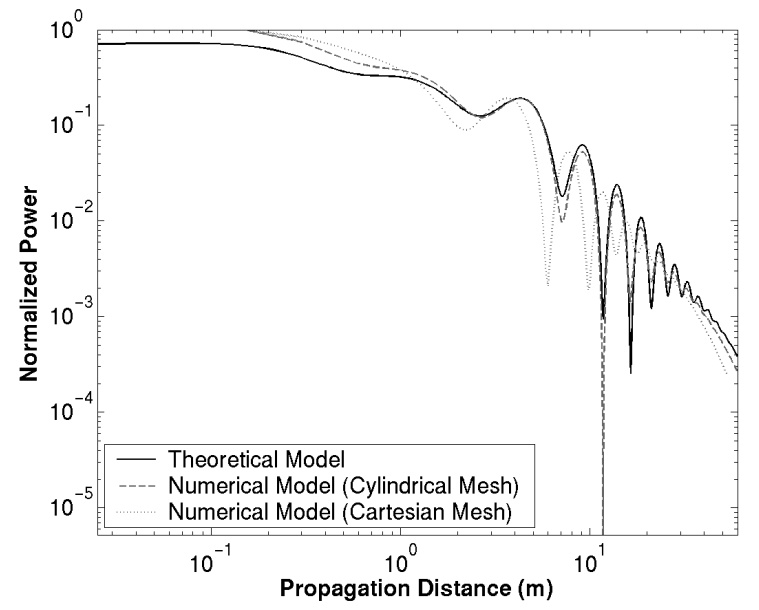

Figure 4: Comparison between theoretical model and two different numerical models at $200 \mathrm{MHz}$ for a smooth cave with conductivity $\sigma_{\text {high }}=0.1 \mathrm{~S} / \mathrm{m}$.

\section{Computational Results}

We now proceed to apply the same process discussed above to the more complicated case of random rough walled caves. The random rough surface is generated as follows. Step 1: Generate a cylindrical surface of appropriate radius and length, Step 2: Add a random perturbation of specified standard deviation to the surface, Step 3: Smooth the random surface (low pass filter) to introduce a surface correlation of a given length, Step 4: Generate a 3D Cartesian mesh, where the electrical conductivity of each element depends upon whether the element is inside the random surface (air) or outside the random surface (earth). For mesh elements that straddle the random surface, a volume-fraction is used to determine the conductivity. To model the dielectric and conducting properties of earth, we choose a uniformly varying electrical conductivity of $0.1-0.02 \mathrm{~S} / \mathrm{m}$ and a dielectric permittivity of 5 
times the free space permittivity $\epsilon_{0}$ (a typical value for granite). Each computational mesh consists of 583, 200 hexahedral elements, an example of which is shown in Figure 5. Note that the portion of the mesh representing the air has been removed to illustrate the random rough surface. The various simulation parameters for the random rough surface computations are summarized in Table 1

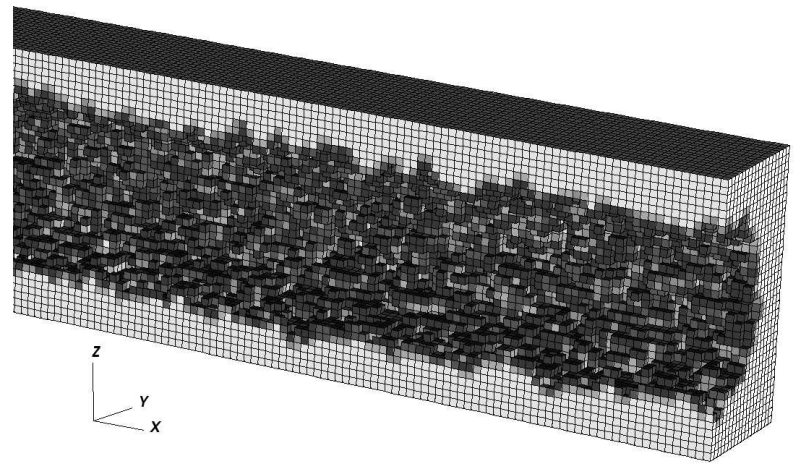

Figure 5: Example of randomly generated cave mesh with interior removed (close-up view).

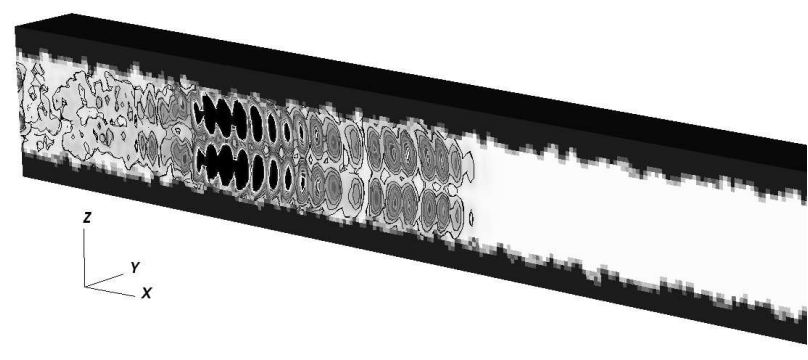

Figure 6: Snapshot of computed electric field magnitude at $t=89.6 \mathrm{ps}$.

\begin{tabular}{l|r}
\hline Avg. Cave Radius & $2 m(1.333 \lambda)$ \\
Cave Length & $75 m(50 \lambda)$ \\
Element Size $(\Delta x)$ & $0.167 m(0.111 \lambda)$ \\
Surface Roughness Variance & $0.3 m(0.2 \lambda)$, Gauss. dist. centered at 0 \\
Conductivity Variation & Uniform, 0.1-0.02 S/m \\
Signal Type & Modulated Gaussian pulse, planar loop antenna \\
Pulse Frequency & $200 \mathrm{MHz}, 20 \%$ Bandwidth \\
Gaussian Width, Delay & $4.67 \mathrm{e}-9 \mathrm{~s}, 2.50 \mathrm{e}-8 \mathrm{~s}$ \\
No. of Trials & 10 \\
No. Unknowns per Trial & $\sim 14$ million \\
No. Parallel CPU's per Trial & 192 \\
\hline
\end{tabular}

Table 1: Summary of computational statistics for 10 random cave simulations.

A total of 10 random caves were simulated. A time history was recorded at each $x$, for all time steps. This data was used to find the spectrum at every spatial step. For each simulation the mean and variance of the power spectral density (PSD) and phase were extracted over the bandwidth of the signal. Each run was normalized by dividing by the total PSD magnitude at the first $x$-data point, thereby removing the characteristics of the input signal, but preserving the relative magnitudes vs. polarization. The last $20 \%$ of the spatial samples were removed to avoid reflections from the end of the cave (due to the imperfect nature of the $\mathrm{ABC}$ ). The results for computed mean power at 5 different frequencies are shown in Figure 7. Note that in general, the scattered field from the rough surface walls fills in nulls which are created by destructive interference in the smooth case (see Figure 3 and Figure 4 for comparrison). In addition, note that the lower frequencies are attenuated more rapidly than the higher frequencies as expected. In Figure 8 we plot the computed variance in power across all 10 simulations.

Figure 8

\section{Conclusions}

We have applied the high order time domain vector finite element methods described in [5], [6] and [7] to the case of RF electric field propagation in a lossy rough wall tunnel. This particular calculation has proved difficult to solve using direct theoretical analysis. We have verified our numerical results by direct comparison to a theoretical model for propagation in a smooth lossy cave. We have presented statistical data for the power spectral density of 


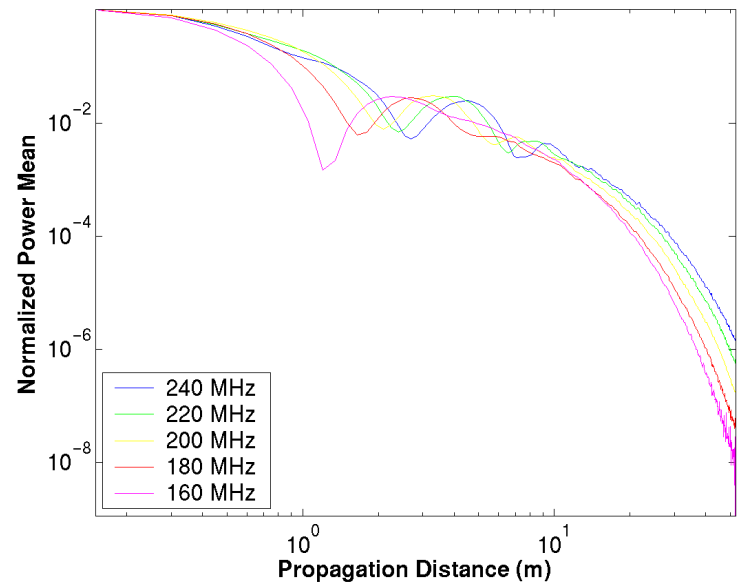

Figure 7: Mean power for 10 random cave simulations at 5 different frequencies.

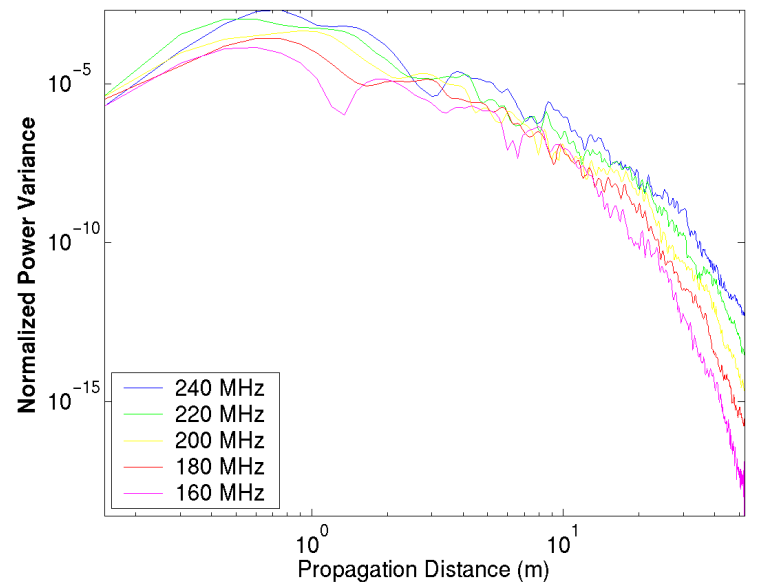

Figure 8: Variance of power for 10 random cave simulations at 5 different frequencies.

a $200 \mathrm{MHz}$ planar loop antenna and have compared our data in a rough walled cave to one with a smooth surface. Further work will allow direct time domain modeling of more complicated cave structures with bends and forks that are too complex for theoretical techniques.

\section{References}

[1] D. G. Dudley. Wireless propagation in circular tunnels. IEEE Trans. Ant. Prop., 53(1):435-441, 2005.

[2] K. Casey. Modal propagation in a circular waveguide with a rough wall. In Proceedings of the 2003 URSI North American Radio Science Meeting, page 534, Columbus, Ohio, June 2003.

[3] H-Y. Pao. Probability-density function for total fields in a straight PEC rough-wall tunnel. Microwave and Opt. Tech. Letters, 46(2):128-132, 2005.

[4] EMSolve - unstructured grid computational electromagnetics using mixed finite element methods. www.llnl.gov/casc/emsolve.

[5] G. Rodrigue and D. White. A vector finite element time-domain method for solving maxwell's equations on unstructured hexahedral grids. SIAM J. Sci. Comp., 23(3):683-706, 2001.

[6] R. Rieben, D. White, and G. Rodrigue. A high order mixed vector finite element method for solving the time dependent Maxwell equations on unstructured grids. J. Comput. Phys., 204:490-519, 2005.

[7] A. Fisher, R. Rieben, G. Rodrigue, and D. White. A generalized mass lumping technique for vector finite element solutions of the time dependent Maxwell equations. IEEE Trans. Ant. Prop., 53(9), 2005. 